\title{
Opial-type inequalities for convex functions and associated results in fractional calculus
}

\author{
G. Farid ${ }^{1 *}$, A.U. Rehman', S. Ullah², A. Nosheen ${ }^{3}$, M. Waseem ${ }^{4}$ and Y. Mehboob
}

\section{"Correspondence:}

faridphdsms@hotmail.com;

ghlmfarid@cuiatk.edu.pk

'Department of Mathematics,

COMSATS University Islamabad

Attock Campus, Pakistan

Full list of author information is

available at the end of the article

\begin{abstract}
This paper is dedicated to Opial-type inequalities for arbitrary kernels using convex functions. These inequalities are further applied to a power function. Applications of the presented results are studied in fractional calculus via fractional integral operators by associating special kernels.
\end{abstract}

MSC: 26A51; 26A33; 33E12

Keywords: Opial inequality; Convex function; Fractional integral operators

\section{Introduction and preliminary results}

Opial obtained the following integral inequality in 1960 [25].

Theorem 1.1 Let $g \in C^{1}[0, h]$ be such that $g(0)=g(h)=0$ and $g(t)>0$ for $t \in(0, h)$. Then

$$
\int_{0}^{h}\left|g(t) g^{\prime}(t)\right| d t \leq \frac{h}{4} \int_{0}^{h}\left(g^{\prime}(t)\right)^{2} d t
$$

Here $\frac{h}{4}$ is a best possible constant.

This inequality has been studied by several mathematicians, and several related inequalities have been investigated. For example its generalizations were published by Beesack in 1962 and 1971 [3, 5], Hua in 1965 [17], Redheffer in 1966 [30], Calvert in 1967 [8], Godunova and Levin in 1967 [13], Maroni in 1967 [22], Boyd and Wong in 1967 [7], Beesack and Das in 1968 [4], Boyd in 1969 [6], Rozanova in 1972 [31], Vrănceanu in 1973 [35], Shum in 1974 and 1975 [32, 33], Hou in 1979 [16], G. Milovanovic and I. Milovanovic in 1980 [23], Lee in 1980 [20], He and Wang in 1981 [14], Yang in 1966 and 1983 [37, 38], Hong, Yang, and Du in 1982 [15], Lin and Yang in 1985 [21], Qi in 1985 [29], Fagbohun and Imoru in 1985 and 1986 [9, 10], Pachpatte in 1986 and 1993 [26, 27], Mitrinović and Pečarić in 1988 [24], Hwang and Yang in 1990 [18], and Sinnamon in 1991 [34].

The aim of this paper is to establish some new Opial-type inequalities for convex functions. Therefore, in the subsequent convex function, its properties, characterization, and Opial-type inequalities for convex functions have been summarized as motivation behind the recent work.

(c) The Author(s) 2019. This article is distributed under the terms of the Creative Commons Attribution 4.0 International License (http://creativecommons.org/licenses/by/4.0/), which permits unrestricted use, distribution, and reproduction in any medium, provided you give appropriate credit to the original author(s) and the source, provide a link to the Creative Commons license, and indicate if changes were made. 
Definition 1 Let $I$ be an interval in $\mathbb{R}$. Then $f: I \rightarrow \mathbb{R}$ is said to be convex if, for all $x, y \in I$ and all $\alpha \in[0,1]$,

$$
f(\alpha x+(1-\alpha) y) \leq \alpha f(x)+(1-\alpha) f(y)
$$

holds.

A characterization of convex function is stated in the following lemma.

Lemma 1.2 ([36]) Let $f$ be a differentiable function on $(a, b)$. Then $f$ is convex if and only if $f^{\prime}$ is an increasing function.

The convexity of composition of two functions can be obtained under the conditions stated in the following lemma.

Lemma 1.3 ([36]) Let $f: I \rightarrow \mathbb{R}$ and $g: J \rightarrow \mathbb{R}$, where range $(f) \subseteq J$. If $f$ and $g$ are convex and $g$ is increasing, then the composite function $g \circ f$ is convex on $I$.

In [24] generalized Opial-type inequalities for convex functions have been proved by Mitrinović and Pečarić. Let $U_{1}(v, k)$ denote the class of functions $u:[a, b] \rightarrow \mathbb{R}$ having representation

$$
u(x)=\int_{a}^{x} k(x, t) v(t) d t,
$$

where $v$ is a continuous function and $k$ is an arbitrary nonnegative kernel such that $k(x, t)=$ 0 for $t>x$, and $v(x)>0$ implies $u(x)>0$ for every $x \in[a, b]$.

Let $U_{2}(v, k)$ denote the class of all the functions $u:[a, b] \rightarrow \mathbb{R}$ having representation

$$
u(x)=\int_{x}^{b} k(x, t) v(t) d t
$$

where $v$ is a continuous function and $k$ is an arbitrary nonnegative kernel such that $k(x, t)=$ 0 for $t<x$, and $v(x)>0$ implies $u(x)>0$ for every $x \in[a, b]$.

Theorem 1.4 ([28]) Let $\phi:[0, \infty) \rightarrow \mathbb{R}$ be a differentiable function such that, for $q>1$, the function $\phi\left(x^{1 / q}\right)$ is convex and $\phi(0)=0$. Let $u \in U_{1}(v, k)$ where $\left(\int_{a}^{x}(k(x, t))^{p} d t\right)^{1 / p} \leq K$ and $\frac{1}{p}+\frac{1}{q}=1$. Then

$$
\int_{a}^{b}|u(x)|^{1-q} \phi^{\prime}(|u(x)|)|v(x)|^{q} d x \leq \frac{q}{K^{q}} \phi\left(K\left(\int_{a}^{b}|v(x)|^{q} d x\right)^{1 / q}\right) .
$$

If the function $\phi\left(x^{1 / q}\right)$ is concave, then the reverse inequality holds.

A similar result was obtained for class $U_{2}(v, k)$.

Theorem 1.5 ([28]) Let $\phi:[0, \infty) \rightarrow \mathbb{R}$ be a differentiable function such that, for $q>1$, the function $\phi\left(x^{1 / q}\right)$ is convex and $\phi(0)=0$. Let $u \in U_{2}(v, k)$ where $\left(\int_{x}^{b}(k(x, t))^{p} d t\right)^{1 / p} \leq K$ and 
$\frac{1}{p}+\frac{1}{q}=1$. Then

$$
\int_{a}^{b}|u(x)|^{1-q} \phi^{\prime}(|u(x)|)|v(x)|^{q} d x \leq \frac{q}{K^{q}} \phi\left(K\left(\int_{a}^{b}|v(x)|^{q} d x\right)^{1 / q}\right) .
$$

If the function $\phi\left(x^{1 / q}\right)$ is concave, then the reverse inequality holds.

In [1] Andrić et al. further extended these results stating the following.

Theorem $1.6([1])$ Let $\phi:[0, \infty) \rightarrow \mathbb{R}$ be a differentiable function such that, for $q>1$, the function $\phi\left(x^{1 / q}\right)$ is convex and $\phi(0)=0$. Let $u \in U_{1}(v, k)$ where $\left(\int_{a}^{x}(k(x, t))^{p} d t\right)^{1 / p} \leq K$ and $\frac{1}{p}+\frac{1}{q}=1$. Then

$$
\begin{aligned}
\int_{a}^{b}|u(x)|^{1-q} \phi^{\prime}(|u(x)|)|v(x)|^{q} d x & \leq \frac{q}{K^{q}} \phi\left(K\left(\int_{a}^{b}|v(x)|^{q} d x\right)^{1 / q}\right) \\
& \leq \frac{q}{K^{q}(b-a)} \int_{a}^{b} \phi\left((b-a)^{1 / q} K|v(x)|\right) d x
\end{aligned}
$$

If the function $\phi\left(x^{1 / q}\right)$ is concave, then the reverse inequality holds.

A similar result for class $U_{2}(v, k)$ is stated in the following theorem.

Theorem $1.7([1])$ Let $\phi:[0, \infty) \rightarrow \mathbb{R}$ be a differentiable function such that, for $q>1$, the function $\phi\left(x^{1 / q}\right)$ is convex and $\phi(0)=0$. Let $u \in U_{2}(v, k)$ where $\left(\int_{x}^{b}(k(x, t))^{p} d t\right)^{1 / p} \leq K$ and $\frac{1}{p}+\frac{1}{q}=1$. Then

$$
\begin{aligned}
\int_{a}^{b}|u(x)|^{1-q} \phi^{\prime}(|u(x)|)|v(x)|^{q} d x & \leq \frac{q}{K^{q}} \phi\left(K\left(\int_{a}^{b}|v(x)|^{q} d x\right)^{1 / q}\right) \\
& \leq \frac{q}{K^{q}(b-a)} \int_{a}^{b} \phi\left((b-a)^{1 / q} K|v(x)|\right) d x
\end{aligned}
$$

If the function $\phi\left(x^{1 / q}\right)$ is concave, then the reverse inequality holds.

In $[11,12]$ Farid and Pečarić studied these inequalities in a fractional point of view. They considered several fractional integral operators via particular kernels to obtain RiemannLiouville, Caputo, and Canavati fractional Opial-type inequalities. Extensions of these Opial-type fractional inequalities have been proved in [1] by Andrić et al., Basci and Dumitru in [2] considered some new aspects of these inequalities.

Next we present the Riemann-Liouville fractional integral, Caputo, and Canavati fractional derivatives [19].

Definition 2 Let $f \in L_{1}[a, b]$. Then the left-sided and right-sided Riemann-Liouville fractional integrals of order $\alpha>0$ with $a \geq 0$ are defined as follows:

$$
I_{a+}^{\alpha} f(x)=\frac{1}{\Gamma(\alpha)} \int_{a}^{x}(x-t)^{\alpha-1} f(t) d t, \quad x>a,
$$


and

$$
I_{b-}^{\alpha} f(x)=\frac{1}{\Gamma(\alpha)} \int_{x}^{b}(t-x)^{\alpha-1} f(t) d t, \quad x<b
$$

where $\Gamma(\cdot)$ is the gamma function.

Definition 3 Let $\alpha>0$ and $\alpha \notin\{1,2,3, \ldots\}, n=[\alpha]+1, f \in A C^{n}[a, b]$. Then the left-sided and right-sided Caputo fractional derivatives of order $\alpha$ are defined as follows:

$$
\left({ }^{C} D_{a+}^{\alpha} f\right)(x)=\frac{1}{\Gamma(n-\alpha)} \int_{a}^{x} \frac{f^{(n)}(t)}{(x-t)^{\alpha-n+1}} d t, \quad x>a,
$$

and

$$
\left({ }^{C} D_{b-}^{\alpha} f\right)(x)=\frac{(-1)^{n}}{\Gamma(n-\alpha)} \int_{x}^{b} \frac{f^{(n)}(t)}{(t-x)^{\alpha-n+1}} d t, \quad x<b .
$$

In [1] composition identities for the Caputo fractional derivatives are given, they are stated in the following lemmas.

Lemma 1.8 Let $\beta>\alpha \geq 0, m=[\beta]+1$, and $n=[\alpha]+1$ for $\alpha, \beta \notin \mathbb{N}_{0} ; n=[\alpha]$ and $m=$ $[\beta]$ for $\alpha, \beta \in \mathbb{N}_{0}$. Let $f \in A C^{m}[a, b]$ be such that $f^{(i)}(a)=0$ for $i=n, n+1, \ldots, m-1$. Let ${ }^{C} D_{a+}^{\beta} f,{ }^{C} D_{a+}^{\alpha} f \in L_{1}[a, b]$. Then

$$
{ }^{C} D_{a+}^{\alpha} f(x)=\frac{1}{\Gamma(\beta-\alpha)} \int_{a}^{x}(x-t)^{\beta-\alpha-1 C} D_{a+}^{\beta} f(t) d t, \quad x \in[a, b] .
$$

Lemma 1.9 Let $\beta>\alpha \geq 0, m=[\beta]+1$, and $n=[\alpha]+1$ for $\alpha, \beta \notin \mathbb{N}_{0} ; n=[\alpha]$ and $m=$ $[\beta]$ for $\alpha, \beta \in \mathbb{N}_{0}$. Let $f \in A C^{m}[a, b]$ be such that $f^{(i)}(b)=0$ for $i=n, n+1, \ldots, m-1$. Let ${ }^{C} D_{b-}^{\alpha} f,{ }^{C} D_{b-}^{\beta} f \in L_{1}[a, b]$. Then

$$
{ }^{C} D_{b-}^{\alpha} f(x)=\frac{1}{\Gamma(\beta-\alpha)} \int_{x}^{b}(t-x)^{\beta-\alpha-1 C} D_{b-}^{\beta} f(t) d t, \quad x \in[a, b] .
$$

Next consider a subspace $C_{a+}^{\alpha}[a, b]$ defined by

$$
C_{a+}^{\alpha}[a, b]=\left\{f \in C^{n-1}[a, b]: I_{a+}^{n-\alpha} f^{(n-1)} \in C^{1}[a, b]\right\} .
$$

Definition 4 Let $f \in C_{a+}^{\alpha}[a, b]$. Then the left-sided Canavati fractional derivative is defined by

$$
{ }^{\tilde{C}} D_{a+}^{\alpha} f(x)=\frac{1}{\Gamma(n-\alpha)} \frac{d}{d x} \int_{a}^{x}(x-t)^{n-\alpha-1} f^{(n-1)}(t) d t=\frac{d}{d x} I_{a+}^{n-\alpha} f^{(n-1)}(x) .
$$

Composition identity for the left-sided Canavati fractional derivative is given in the following lemma. 
Lemma 1.10 ([1]) Let $\beta>\alpha>0, m=[\beta]+1, n=[\alpha]+1$. Let $f \in C_{a+}^{\beta}[a, b]$ be such that $f^{i}(a)=0$ for $i=n-1, n, \ldots, m-2$. Then $f \in C_{a+}^{\alpha}[a, b]$ and

$$
{ }^{\tilde{C}} D_{a+}^{\alpha} f(x)=\frac{1}{\Gamma(\beta-\alpha)} \int_{a}^{x}(x-t)^{\beta-\alpha-1} \tilde{C}^{\beta}{ }_{a+}^{\beta} f(t) d t, \quad x \in[a, b] .
$$

Definition 5 Let $f \in L_{1}[a, b]$. Then the left-sided Riemann-Liouville fractional derivative of order $\alpha$ is defined by

$$
D_{a+}^{\alpha} f(x)=\frac{1}{\Gamma(n-\alpha)} \frac{d^{n}}{d x^{n}} \int_{a}^{x}(x-t)^{n-\alpha-1} f(t) d t=\frac{d^{n}}{d x^{n}} I_{a+}^{n-\alpha} f(x) .
$$

The following lemma summarizes conditions in the composition identity for the leftsided Riemann-Liouville fractional derivative.

Lemma 1.11 ([1]) Let $\beta>\alpha \geq 0, m=[\beta]+1, n=[\alpha]+1$. The composition identity

$$
D_{a+}^{\alpha} f(x)=\frac{1}{\Gamma(\beta-\alpha)} \int_{a}^{x}(x-t)^{\beta-\alpha-1} D_{a+}^{\beta} f(t) d t, \quad x \in[a, b]
$$

is valid if one of the following conditions holds:

(i) $f \in I_{a+}^{\beta}\left(L_{1}[a, b]\right)=\left\{f: f=I_{a+}^{\beta} \varphi, \varphi \in L_{1}[a, b]\right\}$.

(ii) $I_{a+}^{m-\beta} f \in A C^{m}[a, b]$ and $D_{a+}^{\beta-k} f(a)=0$ for $k=1, \ldots, m$.

(iii) $D_{a+}^{\beta-1} f \in A C[a, b], D_{a+}^{\beta-k} f \in C[a, b]$ and $D_{a+}^{\beta-k} f(a)=0$ for $k=1, \ldots, m$.

(iv) $f \in A C^{m}[a, b], D_{a+}^{\beta} f, D_{a+}^{\alpha} f \in L_{1}[a, b], \beta-\alpha \notin \mathbb{N}, D_{a+}^{\beta-k} f(a)=0$ for $k=1, \ldots, m$ and $D_{a+}^{\alpha-k} f(a)=0$ for $k=1, \ldots, n$.

(v) $f \in A C^{m}[a, b], D_{a+}^{\beta} f, D_{a+}^{\alpha} f \in L_{1}[a, b], \beta-\alpha=l \in \mathbb{N}, D_{a+}^{\beta-k} f(a)=0$ for $k=1, \ldots, l$.

(vi) $f \in A C^{m}[a, b], D_{a+}^{\beta} f, D_{a+}^{\alpha} f \in L_{1}[a, b]$ and $f^{k}(a)=0$ for $k=0, \ldots, m-2$.

(vii) $f \in A C^{m}[a, b], D_{a+}^{\beta} f, D_{a+}^{\alpha} f \in L_{1}[a, b], \beta \notin \mathbb{N}$ and $D_{a+}^{\beta-1} f$ is bounded in $a$ neighborhood of $a$.

In the following paragraph, the spaces of functions which have been used in definitions and results are summarized.

The space of all continuous functions whose nth time continuous derivative exists on $[a, b]$ is denoted by $C^{n}[a, b]$, the space of all absolutely continuous functions on $[a, b]$ is denoted by $A C[a, b]$. While $A C^{n}[a, b]$ denotes the space of all the functions $f \in C^{n-1}[a, b]$ with $f^{(n-1)} \in A C[a, b]$ and $L_{p}[a, b], 1 \leq p<\infty$, denotes the space of all Lebesgue measurable functions $f$ for which $|f|^{p}$ is Lebesgue integrable on $[a, b]$.

The paper is organized as follows.

In Sect. 2, new Opial-type inequalities for convex functions are established by applying an arbitrary kernel. Moreover, these inequalities are studied for a power function. Furthermore, in Sect. 3 results of Sect. 2 are analyzed for particular kernels, and fractional integral inequalities of Opial-type are produced by using the definitions and composition identities of Riemann-Liouville fractional integral, Caputo fractional derivative, Canavati fractional derivative. The results for fractional inequalities are obtained by using different forms of the weighted functions and kernels. 


\section{Opial-type inequalities for convex functions}

In this section some new generalized Opial-type inequalities for convex functions are obtained.

Theorem 2.1 Let $\phi, g:[0, \infty) \rightarrow \mathbb{R}$ be differentiable convex and increasing functions with $\phi(g(0))=0$. Also, let $u \in U_{1}(g \circ v, k)$ and $|k(x, t)| \leq K$, where $K$ is a constant. Then the following inequalities hold:

$$
\begin{aligned}
\int_{a}^{b} \phi^{\prime}(g(|u(x)|)) g^{\prime}(|u(x)|)|g \circ v(x)| d x & \leq \frac{1}{K} \phi\left(g\left(K \int_{a}^{b}|g \circ v(t)| d t\right)\right) \\
& \leq \frac{1}{K(b-a)} \int_{a}^{b} \phi(g(K(b-a)|g \circ v(t)|)) d t .
\end{aligned}
$$

Proof As $u \in U_{1}(g \circ v, k)$, therefore, it has the representation $u(x)=\int_{a}^{x} k(x, t) g(v(t)) d t$. By using $|k(x, t)| \leq K$, we find that

$$
|u(x)| \leq K \int_{a}^{x}|g \circ v(t)| d t .
$$

By applying the function $g$ on both sides and using its monotonicity, we get

$$
g(|u(x)|) \leq g\left(K \int_{a}^{x}|g \circ v(t)| d t\right) .
$$

If we set $p(x):=\int_{a}^{x}|g \circ v(t)| d t$, then $p^{\prime}(x)=|g \circ v(x)|$, therefore the last inequality gives $g(|u(x)|) \leq g(K(p(x)))$. As $\phi$ is a differentiable convex function, so by Lemma 1.2, $\phi^{\prime}$ is increasing, therefore we get

$$
\phi^{\prime}(g(|u(x)|)) \leq \phi^{\prime}(g(K p(x))) .
$$

As $g$ is a differentiable convex function, by Lemma 1.2, $g^{\prime}$ is increasing. Using (7), we get

$$
g^{\prime}(|u(x)|) \leq g^{\prime}(K p(x))
$$

From inequalities (8) and (9), the following inequality can be obtained:

$$
\phi^{\prime}(g(|u(x)|)) g^{\prime}(|u(x)|)|g \circ v(x)| \leq \phi^{\prime}(g(K(p(x)))) g^{\prime}(K p(x)) p^{\prime}(x) .
$$

Integrating we have

$$
\int_{a}^{b} \phi^{\prime}(g(|u(x)|)) g^{\prime}(|u(x)|)|\operatorname{gov}(x)| d x \leq \int_{a}^{b} \phi^{\prime}(g(K(p(x)))) g^{\prime}(K p(x)) p^{\prime}(x) d x .
$$

The right-hand side is computed as follows:

$$
\begin{aligned}
& \int_{a}^{b} \phi^{\prime}(g(K p(x))) g^{\prime}(K p(x)) p^{\prime}(x) d x \\
& =\frac{1}{K} \int_{a}^{b} \phi^{\prime}(g(K p(x))) d(g(K p(x)))
\end{aligned}
$$




$$
\begin{aligned}
& =\frac{1}{K} \phi(g(K p(b))) \\
& =\frac{1}{K} \phi\left(g\left(K \int_{a}^{b}|g \circ v(t)| d t\right)\right) .
\end{aligned}
$$

Therefore (11) takes the form as follows:

$$
\int_{a}^{b} \phi^{\prime}(g(|u(x)|)) g^{\prime}(|u(x)|)|\operatorname{gov}(x)| d x \leq \frac{1}{K} \phi\left(g\left(K \int_{a}^{b}|g \circ v(t)| d t\right)\right) .
$$

As $\phi$ and $g$ are convex functions and $\phi$ is an increasing function, so by Lemma 1.3, $\phi \circ g$ is convex and the following Jensen's inequality holds:

$$
\phi\left(g\left(K \int_{a}^{b}|g \circ v(t)| d t\right)\right) \leq \frac{1}{b-a} \int_{a}^{b} \phi(g(K(b-a)|g \circ v(t)|)) d t .
$$

Inequalities (12) and (13) provide the required result.

For power function $g(x)=x^{q}, q \geq 1$, the following Opial-type inequality holds.

Theorem 2.2 Let $\phi:[0, \infty) \rightarrow \mathbb{R}$ be a differentiable convex and increasing function with $\phi(0)=0$. Also let $u \in U_{1}(g \circ v, k)$ and $|k(x, t)| \leq K$, where $K$ is a constant. Then, for $q \geq 1$, the following inequalities hold:

$$
\begin{aligned}
& \int_{a}^{b} \phi^{\prime}\left((|u(x)|)^{q}\right)(|u(x)|)^{q-1}\left|(v(x))^{q}\right| d x \\
& \quad \leq \frac{1}{q K} \phi\left(\left(K \int_{a}^{b}|v(t)|^{q} d t\right)^{q}\right) \\
& \quad \leq \frac{1}{q K(b-a)} \int_{a}^{b} \phi\left(\left(K(b-a)|v(t)|^{q}\right)^{q}\right) d t .
\end{aligned}
$$

Proof Let $g(x)=x^{q}$. Then $g$ is convex and increasing for $q \geq 1$. Therefore applying Theorem 2.1 for the function $g$, inequalities in (6) provide inequalities in (14).

Remark 2.1 Inequalities of Opial-type which are proved in Theorems 2.1 and 2.2 hold too for the class of functions denoted by $U_{2}(g \circ v, k)$. Furthermore, these results for fractional calculus are interesting to see in Theorems 3.2 and 3.4 for right-sided Riemann-Liouville fractional integrals. For Caputo and Canavati derivatives, these results have been comprised in Theorems 3.6 and 3.8.

\section{Fractional Opial-type inequalities}

Here we utilize Riemann-Liouville fractional integrals, Caputo fractional derivatives, composition identities for Caputo fractional derivatives, and composition identities for Canavati fractional derivatives to obtain corresponding fractional Opial-type integral inequalities. 
Theorem 3.1 Let $\phi, g:[0, \infty) \rightarrow \mathbb{R}$ be differentiable convex and increasing functions with $\phi(g(0))=0$. Also let $v \in L[a, b]$. Then, for $\alpha \geq 1$, the following fractional inequalities hold:

$$
\begin{aligned}
& \int_{a}^{b} \phi^{\prime}\left(g\left(\left|I_{a+}^{\alpha} v(x)\right|\right)\right) g^{\prime}\left(\left|I_{a+}^{\alpha} v(x)\right|\right)|g \circ v(x)| d x \\
& \quad \leq \frac{\Gamma(\alpha)}{(b-a)^{\alpha-1}} \phi\left(g\left(\frac{(b-a)^{\alpha-1}}{\Gamma(\alpha)} \int_{a}^{b}|g \circ v(t)| d t\right)\right) \\
& \quad \leq \frac{\Gamma(\alpha)}{(b-a)^{\alpha}} \int_{a}^{b} \phi\left(g\left(\frac{(b-a)^{\alpha}}{\Gamma(\alpha)}|g \circ v(t)|\right)\right) d t .
\end{aligned}
$$

Proof Let us define for $x \in[a, b]$ the kernel $k(x, t)$ as follows:

$$
k(x, t)= \begin{cases}\frac{1}{\Gamma(\alpha)}(x-t)^{\alpha-1}, & a \leq t \leq x, \\ 0, & x<t \leq b .\end{cases}
$$

Also, if $u$ is defined by

$$
u(x)=I_{a+}^{\alpha} v(x)=\frac{1}{\Gamma(\alpha)} \int_{a}^{x}(x-t)^{\alpha-1} v(t) d t
$$

then we have $|k(x, t)| \leq \frac{(x-a)^{\alpha-1}}{\Gamma(\alpha)}$. Further $(x-a)^{\alpha}, \alpha \geq 0$, is increasing on $[a, b]$, therefore we have $|k(x, t)| \leq \frac{(b-a)^{\alpha-1}}{\Gamma(\alpha)}$ for $\alpha \geq 1$. Applying Theorem 2.1 for this particular kernel, we get inequalities in (15).

A similar result can be obtained for the class of functions denoted by $U_{2}(g \circ v, k)$ for the right-sided Riemann-Liouville fractional integral which is stated as follows.

Theorem 3.2 Let $\phi, g:[0, \infty) \rightarrow \mathbb{R}$ be differentiable convex and increasing functions with $\phi(g(0))=0$. Also let $v \in L[a, b]$. Then, for $\alpha \geq 1$, the following fractional inequalities hold:

$$
\begin{aligned}
& \int_{a}^{b} \phi^{\prime}\left(g\left(\left|I_{b-}^{\alpha} v(x)\right|\right)\right) g^{\prime}\left(\left|I_{b-}^{\alpha} v(x)\right|\right)|g \circ v(x)| d x \\
& \quad \leq \frac{\Gamma(\alpha)}{(b-a)^{\alpha-1}} \phi\left(g\left(\frac{(b-a)^{\alpha-1}}{\Gamma(\alpha)} \int_{a}^{b}|g \circ v(t)| d t\right)\right) \\
& \quad \leq \frac{\Gamma(\alpha)}{(b-a)^{\alpha}} \int_{a}^{b} \phi\left(g\left(\frac{(b-a)^{\alpha}}{\Gamma(\alpha)}|g \circ v(t)|\right)\right) d t .
\end{aligned}
$$

Proof The proof is similar to the proof of Theorem 3.1.

Theorem 3.3 Let $\phi:[0, \infty) \rightarrow \mathbb{R}$ be a differentiable convex and increasing function with $\phi(0)=0$. Also let $v \in L[a, b]$. Then, for $\alpha, q \geq 1$, the following fractional inequalities hold:

$$
\begin{aligned}
& \int_{a}^{b} \phi^{\prime}\left(\left|I_{a+}^{\alpha} v(x)\right|^{q}\right)\left|I_{a+}^{\alpha} v(x)\right|^{q-1}|v(x)|^{q} d x \\
& \quad \leq \frac{\Gamma(\alpha)}{q(b-a)^{\alpha-1}} \phi\left(\left(\frac{(b-a)^{\alpha-1}}{\Gamma(\alpha)} \int_{a}^{b}|v(t)|^{q} d t\right)^{q}\right) \\
& \quad \leq \frac{\Gamma(\alpha)}{q(b-a)^{\alpha}} \int_{a}^{b} \phi\left(\left(\frac{(b-a)^{\alpha}}{\Gamma(\alpha)}|v(t)|^{q}\right)^{q}\right) d t .
\end{aligned}
$$


Proof Let $g(x)=x^{q}$. Then $g$ is convex and increasing for $q \geq 1$. Therefore, using this power function $g$ in inequalities (15), we get inequalities in (18).

A similar result for right-sided Riemann-Liouville fractional integrals holds.

Theorem 3.4 Let $\phi:[0, \infty) \rightarrow \mathbb{R}$ be a differentiable convex and increasing function with $\phi(0)=0$. Also let $v \in L[a, b]$. Then, for $\alpha, q \geq 1$, the following inequalities hold:

$$
\begin{aligned}
& \int_{a}^{b} \phi^{\prime}\left(\left|I_{b-}^{\alpha} v(x)\right|^{q}\right)\left|I_{b-}^{\alpha} v(x)\right|^{q-1}|v(x)|^{q} d x \\
& \quad \leq \frac{\Gamma(\alpha)}{q(b-a)^{\alpha-1}} \phi\left(\left(\frac{(b-a)^{\alpha-1}}{\Gamma(\alpha)} \int_{a}^{b}|v(t)|^{q} d t\right)^{q}\right) \\
& \quad \leq \frac{\Gamma(\alpha)}{q(b-a)^{\alpha}} \int_{a}^{b} \phi\left(\left(\frac{(b-a)^{\alpha}}{\Gamma(\alpha)}|v(t)|^{q}\right)^{q}\right) d t
\end{aligned}
$$

Proof The proof is similar to the proof of Theorem 3.3.

For composition identity of left-sided Caputo fractional derivative given in Lemma 1.8, the following result holds.

Theorem 3.5 Let $\phi, g:[0, \infty) \rightarrow \mathbb{R}$ be differentiable convex and increasing functions with $\phi(g(0))=0$. Also let $m=[\beta]+1$ and $n=[\alpha]+1$ for $\alpha, \beta \notin \mathbb{N}_{0} ; n=[\alpha]$ and $m=[\beta]$ for $\alpha, \beta \in$ $\mathbb{N}_{0}$ and $v \in A C^{m}[a, b]$ such that $f^{(i)}(a)=0$ for $i=n, n+1, \ldots, m-1$. Let ${ }^{C} D_{a+}^{\beta} v \in L_{q}[a, b]$ and ${ }^{C} D_{a+}^{\alpha} v \in L_{1}[a, b]$. Then, for $\alpha<\beta-1$, the following fractional inequalities hold:

$$
\begin{aligned}
& \int_{a}^{b} \phi^{\prime}\left(g\left(\left|{ }^{C} D_{a+}^{\alpha} v(x)\right|\right)\right) g^{\prime}\left(\left|{ }^{C} D_{a+}^{\alpha} v(x)\right|\right)\left|g \circ{ }^{C} D_{a+}^{\beta} v(x)\right| d x \\
& \quad \leq \frac{\Gamma(\beta-\alpha)}{(b-a)^{\beta-\alpha-1} \phi\left(g\left(\frac{(b-a)^{\beta-\alpha-1}}{\Gamma(\beta-\alpha)} \int_{a}^{b}\left|g \circ{ }^{C} D_{a+}^{\beta} v(t)\right| d t\right)\right)} \\
& \quad \leq \frac{\Gamma(\beta-\alpha)}{(b-a)^{\beta-\alpha}} \int_{a}^{b} \phi\left(g\left(\frac{(b-a)^{\beta-\alpha}}{\Gamma(\beta-\alpha)}\left|g \circ{ }^{C} D_{a+}^{\beta} v(t)\right|\right)\right) d t .
\end{aligned}
$$

Proof Let us define the kernel $k(x, t)$ for $x \in[a, b]$ as follows:

$$
k(x, t)= \begin{cases}\frac{1}{\Gamma(\beta-\alpha)}(x-t)^{\beta-\alpha-1}, & a \leq t \leq x \\ 0, & x \leq t \leq b\end{cases}
$$

Also let us define the function $u$ by

$$
u(x)={ }^{C} D_{a+}^{\alpha} v(x)=\frac{1}{\Gamma(\beta-\alpha)} \int_{a}^{x}(x-t)^{\beta-\alpha-1 C} D_{a+}^{\beta} v(t) d t
$$

then we have $|k(x, t)| \leq \frac{(x-a)^{\beta-\alpha-1}}{\Gamma(\beta-\alpha)}$ for $\alpha<\beta-1$. Also, for $\alpha<\beta-1,(x-a)^{\beta-\alpha}$ is an increasing function on $[a, b]$, therefore

$$
\left|\int_{a}^{x} k(x, t) d t\right| \leq \frac{(b-a)^{\beta-\alpha}}{\Gamma(\beta-\alpha)}=K
$$

Hence, by applying Theorem 2.1, inequalities in (20) can be obtained. 
A similar result can be obtained from composition identity for right-sided Caputo fractional derivative given in Lemma 1.9, for the class of functions denoted by $U_{2}(g \circ v, k)$.

Theorem 3.6 Let $\phi, g:[0, \infty) \rightarrow \mathbb{R}$ be differentiable convex and increasing functions with $\phi(g(0))=0$. Also let $m=[\beta]+1$ and $n=[\alpha]+1$ for $\alpha, \beta \notin \mathbb{N}_{0} ; n=[\alpha]$ and $m=[\beta]$ for $\alpha, \beta \in$ $\mathbb{N}_{0}$ and $v \in A C^{m}[a, b]$ such that $f^{(i)}(b)=0$ for $i=n, n+1, \ldots, m-1$. Let ${ }^{C} D_{b-}^{\beta} v \in L_{q}[a, b]$ and ${ }^{C} D_{b-}^{\alpha} v \in L_{1}[a, b]$. Then, for $\alpha<\beta-1$, the following fractional inequalities hold:

$$
\begin{aligned}
& \int_{a}^{b} \phi^{\prime}\left(g\left(\left|{ }^{C} D_{b-}^{\alpha} v(x)\right|\right)\right) g^{\prime}\left(\left|{ }^{C} D_{b-}^{\alpha} v(x)\right|\right)\left|g \circ{ }^{C} D_{b_{-}}^{\beta} v(x)\right| d x \\
& \quad \leq \frac{\Gamma(\beta-\alpha)}{(b-a)^{\beta-\alpha-1}} \phi\left(g\left(\frac{(b-a)^{\beta-\alpha-1}}{\Gamma(\beta-\alpha)} \int_{a}^{b}\left|g \circ{ }^{C} D_{b-}^{\beta} v(t)\right| d t\right)\right) \\
& \quad \leq \frac{\Gamma(\beta-\alpha)}{(b-a)^{\beta-\alpha}} \int_{a}^{b} \phi\left(g\left(\frac{(b-a)^{\beta-\alpha}}{\Gamma(\beta-\alpha)}\left|g \circ{ }^{C} D_{b-}^{\beta} v(t)\right|\right)\right) d t .
\end{aligned}
$$

Proof The proof is similar to the proof of Theorem 3.5.

Theorem 3.7 Let $\phi:[0, \infty) \rightarrow \mathbb{R}$ be a differentiable convex and increasing function with $\phi(0)=0$. Also let $m=[\beta]+1$ and $n=[\alpha]+1$ for $\alpha, \beta \notin \mathbb{N}_{0} ; n=[\alpha]$ and $m=[\beta]$ for $\alpha, \beta \in \mathbb{N}_{0}$ and $v \in A C^{m}[a, b]$ such that $f^{(i)}(a)=0$ for $i=n, n+1, \ldots, m-1$. Let ${ }^{C} D_{a+}^{\beta} v \in L_{q}[a, b]$ and ${ }^{C} D_{a+}^{\alpha} \nu \in L_{1}[a, b]$. Then, for $\alpha<\beta-1$ and $q \geq 1$, the following fractional inequalities hold:

$$
\begin{aligned}
& \int_{a}^{b} \phi^{\prime}\left(\left|{ }^{C} D_{a+}^{\alpha} v(x)\right|^{q}\right)\left|{ }^{C} D_{a+}^{\alpha} v(x)\right|^{q-1}\left|{ }^{C} D_{a+}^{\beta} v(x)\right|^{q} d x \\
& \quad \leq \frac{\Gamma(\beta-\alpha)}{q(b-a)^{\beta-\alpha-1}} \phi\left(\left(\frac{(b-a)^{\beta-\alpha-1}}{\Gamma(\beta-\alpha)} \int_{a}^{b}\left|{ }^{C} D_{a+}^{\beta} \nu(t)\right|^{q} d t\right)^{q}\right) \\
& \quad \leq \frac{\Gamma(\beta-\alpha)}{q(b-a)^{\beta-\alpha}} \int_{a}^{b} \phi\left(\left(\frac{(b-a)^{\beta-\alpha}}{\Gamma(\beta-\alpha)}\left|{ }^{C} D_{a+}^{\beta} \nu(t)\right|^{q}\right)^{q}\right) d t .
\end{aligned}
$$

Proof Let $g(x)=x^{q}$. Then $g$ is convex and increasing for $q \geq 1$. Therefore, using this power function $g$ in inequalities (20), we get inequalities in (23).

A similar result can be obtained for the class of functions denoted by $U_{2}(g \circ v, k)$.

Theorem 3.8 Let $\phi:[0, \infty) \rightarrow \mathbb{R}$ be a differentiable convex and increasing function with $\phi(0)=0$. Also let $m=[\beta]+1$ and $n=[\alpha]+1$ for $\alpha, \beta \notin \mathbb{N}_{0} ; n=[\alpha]$ and $m=[\beta]$ for $\alpha, \beta \in \mathbb{N}_{0}$ and $v \in A C^{m}[a, b]$ such that $f^{(i)}(b)=0$ for $i=n, n+1, \ldots, m-1$. Let ${ }^{C} D_{b-}^{\beta} v \in L_{q}[a, b]$ and ${ }^{C} D_{b-}^{\alpha} v \in L_{1}[a, b]$. Then, for $\alpha<\beta-1$ and $q \geq 1$, the following fractional inequalities hold:

$$
\begin{aligned}
& \int_{a}^{b} \phi^{\prime}\left(\left|{ }^{C} D_{b-}^{\alpha} v(x)\right|^{q}\right)\left|{ }^{C} D_{b-}^{\alpha} v(x)\right|^{q-1}\left|{ }^{C} D_{b_{-}}^{\beta} v(x)\right|^{q} d x \\
& \quad \leq \frac{\Gamma(\beta-\alpha)}{q(b-a)^{\beta-\alpha-1}} \phi\left(\left(\frac{(b-a)^{\beta-\alpha-1}}{\Gamma(\beta-\alpha)} \int_{a}^{b}\left|{ }^{C} D_{b-}^{\beta} v(t)\right|^{q} d t\right)^{q}\right) \\
& \quad \leq \frac{\Gamma(\beta-\alpha)}{q(b-a)^{\beta-\alpha}} \int_{a}^{b} \phi\left(\left(\frac{(b-a)^{\beta-\alpha}}{\Gamma(\beta-\alpha)}\left|{ }^{C} D_{b-}^{\beta} v(t)\right|^{q}\right)^{q}\right) d t .
\end{aligned}
$$


Proof The proof is similar to the proof of Theorem 3.7.

Next result includes the Canavati fractional derivatives using composition identity given in Lemma 1.10.

Theorem 3.9 Let $\phi, g:[0, \infty) \rightarrow \mathbb{R}$ be differentiable convex and increasing functions with $\phi(g(0))=0$. Also let $\alpha \geq 0, m=[\beta]+1$ and $n=[\alpha]+1$. Let $v \in C_{a_{+}}^{\beta}[a, b]$ such that $f^{(i)}(a)=0$ for $i=n-1, n, \ldots, m-2$. Let ${ }^{\tilde{C}} D_{a+}^{\beta} v \in L_{q}[a, b]$. Then, for $\alpha<\beta-1$, the following fractional inequalities hold:

$$
\begin{aligned}
& \int_{a}^{b} \phi^{\prime}\left(g\left(\left|{ }^{\tilde{C}} D_{a_{+}}^{\alpha} v(x)\right|\right)\right) g^{\prime}\left(\left|{ }^{\tilde{C}} D_{a_{+}}^{\alpha} v(x)\right|\right)\left|g \circ{ }^{\tilde{C}} D_{a_{+}}^{\beta} \nu(x)\right| d x \\
& \quad \leq \frac{\Gamma(\beta-\alpha)}{(b-a)^{\beta-\alpha-1}} \phi\left(g\left(\frac{(b-a)^{\beta-\alpha-1}}{\Gamma(\beta-\alpha)} \int_{a}^{b}\left|g \circ{ }^{\tilde{C}} D_{a_{+}}^{\beta} v(t)\right| d t\right)\right) \\
& \quad \leq \frac{\Gamma(\beta-\alpha)}{q(b-a)^{\beta-\alpha}} \int_{a}^{b} \phi\left(g\left(\frac{(b-a)^{\beta-\alpha}}{\Gamma(\beta-\alpha)}\left|g \circ{ }^{\tilde{C}} D_{a_{+}}^{\beta} v(t)\right|\right)\right) d t .
\end{aligned}
$$

Theorem 3.10 Let $\phi:[0, \infty) \rightarrow \mathbb{R}$ be a differentiable convex and increasing function with $\phi(0)=0$. Also let $\alpha \geq 0, m=[\beta]+1$, and $n=[\alpha]+1$. Let $v \in C_{a+}^{\beta}[a, b]$ such that $f^{(i)}(a)=0$ for $i=n-1, n, \ldots, m-2$. Let ${ }^{\tilde{C}} D_{a+}^{\beta} \nu \in L_{q}[a, b]$. Then, for $\alpha<\beta-1$ and $q \geq 1$, the following fractional inequalities hold:

$$
\begin{aligned}
& \int_{a}^{b} \phi^{\prime}\left(\left|\tilde{C}^{\tilde{C}} D_{a+}^{\alpha} \nu(x)\right|^{q}\right)\left|{ }^{\tilde{C}} D_{a+}^{\alpha} \nu(x)\right|^{q-1}\left|\tilde{C}^{\tilde{C}} D_{a+}^{\beta} \nu(x)\right|^{q} d x \\
& \quad \leq \frac{\Gamma(\beta-\alpha)}{q(b-a)^{\beta-\alpha-1}} \phi\left(\left(\left.\left.\frac{(b-a)^{\beta-\alpha-1}}{\Gamma(\beta-\alpha)} \int_{a}^{b}\right|^{\tilde{C}} D_{a+}^{\beta} \nu(t)\right|^{q} d t\right)^{q}\right) \\
& \quad \leq \frac{\Gamma(\beta-\alpha)}{q(b-a)^{\beta-\alpha}} \int_{a}^{b} \phi\left(\left(\frac{(b-a)^{\beta-\alpha}}{\Gamma(\beta-\alpha)}\left|\tilde{C}^{\beta} D_{a+}^{\beta} \nu(t)\right|^{q}\right)^{q}\right) d t .
\end{aligned}
$$

Next results are for Riemann-Liouville fractional derivatives using the composition identity given in Lemma 1.11 .

Theorem 3.11 Let $\phi, g:[0, \infty) \rightarrow \mathbb{R}$ be differentiable convex and increasing functions with $\phi(g(0))=0$. Also let $\alpha \geq 0, m=[\beta]+1$, and $n=[\alpha]+1$. Suppose that one of the following conditions (i)-(vii) in Lemma 1.11 holds for $\{\beta, \alpha, v\}$, and let $D_{a+}^{\beta} v \in L_{q}[a, b]$. Then, for $\alpha<$ $\beta-1$, the following fractional inequalities hold:

$$
\begin{aligned}
& \int_{a}^{b} \phi^{\prime}\left(g\left(\left|D_{a_{+}}^{\alpha} v(x)\right|\right)\right) g^{\prime}\left(\left|D_{a_{+}}^{\alpha} v(x)\right|\right)\left|g \circ D_{a^{+}}^{\beta} v(x)\right| d x \\
& \quad \leq \frac{\Gamma(\beta-\alpha)}{q(b-a)^{\beta-\alpha-1}} \phi\left(g\left(\frac{(b-a)^{\beta-\alpha-1}}{\Gamma(\beta-\alpha)} \int_{a}^{b}\left|g \circ D_{a^{\prime}}^{\beta} v(t)\right| d t\right)\right) \\
& \quad \leq \frac{\Gamma(\beta-\alpha)}{(b-a)^{\beta-\alpha}} \int_{a}^{b} \phi\left(g\left(\frac{(b-a)^{\beta-\alpha}}{\Gamma(\beta-\alpha)}\left|g \circ D_{a_{+}}^{\beta} v(t)\right|\right)\right) d t .
\end{aligned}
$$

Theorem 3.12 Let $\phi:[0, \infty) \rightarrow \mathbb{R}$ be a differentiable convex and increasing function with $\phi(0)=0$. Also let $\alpha \geq 0, m=[\beta]+1$, and $n=[\alpha]+1$. Suppose that one of the following 
conditions (i)-(vii) in Lemma 1.11 hold for $\{\beta, \alpha, v\}$, and let $D_{a+}^{\beta} v \in L_{q}[a, b]$. Then, for $\alpha<$ $\beta-1$ and $q \geq 1$, the following fractional inequalities hold:

$$
\begin{aligned}
& \int_{a}^{b} \phi^{\prime}\left(\left|D_{a_{+}}^{\alpha} \nu(x)\right|^{q}\right)\left|D_{a_{+}}^{\alpha} \nu(x)\right|^{q-1}\left|D_{a_{+}}^{\beta} v(x)\right|^{q} d x \\
& \quad \leq \frac{\Gamma(\beta-\alpha)}{q(b-a)^{\beta-\alpha-1}} \phi\left(\left(\frac{(b-a)^{\beta-\alpha-1}}{\Gamma(\beta-\alpha)} \int_{a}^{b}\left|D_{a+}^{\beta} v(t)\right|^{q} d t\right)^{q}\right) \\
& \quad \leq \frac{\Gamma(\beta-\alpha)}{q(b-a)^{\beta-\alpha}} \int_{a}^{b} \phi\left(\left(\frac{(b-a)^{\beta-\alpha}}{\Gamma(\beta-\alpha)}\left|D_{a_{+}}^{\beta} \nu(t)\right|^{q}\right)^{q}\right) d t .
\end{aligned}
$$

\section{Concluding remarks}

The aim of this paper is to utilize the classes of functions $U_{i}(g \circ v, k), i=1,2$, in the establishment of new Opial-type inequalities in general prospect for convex functions. A power function is considered to obtain particular Opial-type inequalities. In application point of view, all these results have been discussed for fractional calculus operators of RiemannLiouville, Caputo, and Canavati. Fractional inequalities have wide applications in the theory of fractional differential equations and boundary value problems and other modern areas of science like rheology, viscoelasticity, acoustics, optics, chemical and statistical physics, robotics, control theory, electrical and mechanical engineering, bioengineering, etc.

\section{Acknowledgements \\ We thank the editor and referees for their careful reading and valuable suggestions to make the article reader friendly. The research work of Ghulam Farid is supported by the Higher Education Commission of Pakistan under NRPU 2016, Project.}

\section{Funding}

Not applicable.

\section{Competing interests}

It is declared that authors have no competing interests.

\section{Authors' contributions}

All authors have equal contributions in this article. All authors read and approved the final manuscript.

\section{Author details}

'Department of Mathematics, COMSATS University Islamabad, Attock Campus, Pakistan. ${ }^{2}$ Department of Mathematics, Air University, Islamabad, Pakistan. ${ }^{3}$ Department of Mathematics, University of Lahore (Sargodha Campus), Sargodha,

Pakistan. ${ }^{4}$ Department of Mathematics, COMSATS University Islamabad, Vehari Campus, Pakistan.

\section{Publisher's Note}

Springer Nature remains neutral with regard to jurisdictional claims in published maps and institutional affiliations.

Received: 11 January 2019 Accepted: 9 April 2019 Published online: 24 April 2019

\section{References}

1. Andrić, M., Barbir, A., Farid, G., Pečarić, J.: Opial-type inequality due to Agarwal-Pang and fractional differential inequalities. Integral Transforms Spec. Funct. 25(4), 324-335 (2013)

2. Basci, Y., Dumitru, D.: New aspects of Opial-type integral inequalities. Adv. Differ. Equ. 2018, 452 (2018), https://doi.org/10.1186/s13662-018-1912-4

3. Beesack, P.R.: Integral inequalities involving a function and its derivative. Am. Math. Mon. 78, 705-741 (1971)

4. Beesack, P.R., Das, K.M.: Extensions of Opial's inequality. Pac. J. Math. 26, 215-232 (1968)

5. Beesack, R.P.: On and integral inequality of Z. Opial. Trans. Am. Math. Soc. 104, 470-475 (1962)

6. Boyd, D.W.: Best constants in a class of integral inequalities. Pac. J. Math. 30, 367-383 (1969)

7. Boyd, D.W., Wong, J.S.W.: An extension of Opial's inequality. J. Math. Anal. Appl. 19, 100-102 (1967)

8. Calvert, J.: Some generalization of Opial's inequality. Proc. Am. Math. Soc. 18, 72-75 (1967)

9. Fagbohun, A.B., Imoru, C.O.: On an extension of Opial's inequalities. Simon Stevin 59, 11-19 (1986)

10. Fagbohun, A.B., Imoru, C.O.: A new class of integro-differential inequalities. Simon Stevin 60, 301-311 (1986)

11. Farid, G., Pečarić, J.: Opial type integral inequalities for fractional derivatives. Fract. Differ. Calc. 2(1), 31-54 (2012) 
2. Farid, G., Pečarić, J.: Opial type integral inequalities for fractional derivatives II. Fract. Differ. Calc. 2(2), 139-155 (2012)

13. Godunova, E.K., Levin, V.I.: On an inequality of Maroni (Russian). Mat. Zametki 2, 221-224 (1967)

14. He, T.X., Wang, S.C.: A note on the Opial-Hua inequality (Chinese). J. Math. Res. Exposition 1,61-62 (1981)

15. Hong, Y., Yang, H., Du, D.: An inequality for convex functions. Chin. Sci. Bull. 27, 1266-1270 (1982)

16. Hou, M.S.: An inequality of Z. Opial (Chinese). Chin. Sci. Bull. 24, 247-248 (1979)

17. Hua, L.K.: On an inequality of Opial. Sci. Sin. 14, 789-790 (1965)

18. Hwang, T.S., Yang, G.S.: On integral inequalities related to Opial's inequality. Tamkang J. Math. 21, 177-183 (1990)

19. Kilbas, A.A., Srivastava, H.M., Trujillo, J.J.: Theory and Applications of Fractional Differential Equations. North-Holland Mathematics Studies, vol. 204. Elsevier, New York (2006)

20. Lee, C.S.: On some generalization of inequalities of Opial, Yang and Shum. Can. Math. Bull. 23, 71-80 (1980)

21. Lin, C.T., Yang, G.S.: On some integro-differential inequalities. Tamkang J. Math. 16, 123-129 (1985)

22. Maroni, P.M.: Sur l'inegalite d'Opial-Beesack. C. R. Acad. Sci. Paris, Ser. A 264, 62-64 (1967)

23. Milovanovic, G.V., Milovanovic, I.Z.: The best constant in some integral inequalities of Opial type. Publ. Elektroteh. Fak. Univ. Beogr., Ser. Mat. Fiz. 678(715), 48-53 (1980)

24. Mitrinović, D.S., Pečarić, J.E.: Generalization of two inequalities of Godunova and Levin. Bull. Pol. Acad. Sci., Math. 36 645-648 (1988)

25. Opial, Z:: Sur une inégalité. Ann. Pol. Math. 8, 29-32 (1960)

26. Pachpatte, B.G.: On Opial-type integral inequalities. J. Math. Anal. Appl. 120, 547-556 (1986)

27. Pachpatte, B.G.: A note on generalization Opial type inequalities. Tamkang J. Math. 24, 229-235 (1993)

28. Pečarić, J.E., Proschan, F., Tong, Y.L.: Convex Functions, Partial Orderings, and Statistical Applications. Mathematics in Science and Engineering, vol. 187 (1992)

29. Qi, Z:: Further generalization of Opial's inequality. Acta Math. Sin. New Ser. 1, 196-200 (1985)

30. Redheffer, R.: Inequalities with three functions. J. Math. Anal. Appl. 16, 219-242 (1966)

31. Rozanova, G.l.: Integral'nye neravenstva s proizvodnysi i proizvol'nymivypuklymi funkcijami. Uc. Zap. Mosk. Gos. Ped. In-ta im. Lenina 460, 58-65 (1972)

32. Shum, D.T.: A general and sharpened form of Opial's inequality. Can. Math. Bull. 17, 385-389 (1974)

33. Shum, D.T.: On a class of new inequalities. Trans. Am. Math. Soc. 204, 299-341 (1975)

34. Sinnamon, G.J.: Weighted Hardy and Opial-type inequalities. J. Math. Anal. Appl. 160, 434-445 (1991)

35. Vranceanu, G.G.: On an inequality of Opial. Bull. Math. Soc. Sci. Math. R. S. Roumaine 17, 315-316 (1973)

36. Wayne, A., Varberg, D.E.: Convex Functions. Acadamic Press, New York (1973)

37. Yang, G.S.: On a certain result of Z. Opial. Proc. Jpn. Acad. 42, 78-83 (1966)

38. Yang, G.S.: A note on some integro-differential inequalities. Soochow J. Math. 9, 231-236 (1983)

\section{Submit your manuscript to a SpringerOpen ${ }^{\circ}$ journal and benefit from:}

- Convenient online submission

- Rigorous peer review

- Open access: articles freely available online

- High visibility within the field

- Retaining the copyright to your article

Submit your next manuscript at $\gg$ springeropen.com 